\title{
Análise de inversão para modelos altamente fraturados
}

Thiago P. Prestes* (Universidade Federal do Pará, Brasil), Léo Kirchhof Santos (Universidade Federal do Pará, Brasil) e José J. S. de Figueiredo (Universidade Federal do Pará, Brasil \& INCT-GP, Brasil)

Copyright 2018, SBGf - Sociedade Brasileira de Geofísica

This paper was prepared for presentation during the $8^{\text {th }}$ Brazilian Symposium on Geophysics, held in Salinópolis, Brazil, 18 to 20 setember 2018.

Contents of this paper were reviewed by the Technical Committee of the $8^{\text {th }}$ Brazilian Symposium on Geophysics and do not necessarily represent any position of the SBGf, its officers or members. Electronic reproduction or storage of any part of this paper for commercial purposes without the written consent of The Brazilian Geophysical Society is prohibited.

\section{Abstract}

The knowledge about properties of fractures in subsurface is very important for the oil and gas industry since fractured reservoirs are, in some, the main conduit for hydrocarbon flow. One way to indirectly study the properties of rocks is using the effective medium theories, which are mathematical equations that represent the influence of physical and petrophysical properties of rocks in the seismic waves.

In this work, two models, created by Hudson and Liu (1999), for effective elastic properties of rocks with an array of parallel faults based on the averaging process of Schoenberg and Douma (1988) were studied. In these models, the behavior of the velocities of the $P$ and $S$ waves was analyzed. Depending on the direction of propagation and the polarization of the seismic waves, their velocities have a greater or lesser influence due to the distance between the cracks or total crack density.

\section{Introdução}

Fraturas são discontinuidades existentes em rochas e são amplamente distribuídas na subsuperfície da Terra, tendo grande variedade de tamanhos, orientações, entre outras propriedades. Elas são criadas quando a tensão que age na rocha é maior que sua resistência. Em alguns reservatórios, as fraturas são os principais condutos para fluxo de hidrocarboneto. Portanto, é altamente importante estudar e caracterizar as fraturas em subsuperfície com o objetivo de otimizar a produção de hidrocarbonetos em um processo exploratório (Nelson, 1985; Sayers, 2007).

Uma maneira de analisar o comportamento de ondas sísmicas para diferentes situações geológicas é utilizando teorias de meios efetivos (Hudson, 1980, 1981; Hudson and Liu, 1999). Elas são equações que expressam as mudanças nas propriedades de ondas sísmicas em termos das propriedades do meio por onde se propagam. No estudo de meios fraturados, as teorias de meios efetivos expressam, por exemplo, como as velocidades de ondas $P$ e $\mathrm{S}$ e/ou os parâmetros anisotrópicos a elas associados variam em função das propriedades de fraturas, como forma, densidade e fluido saturante.

O objetivo do trabalho é fazer uma análise do modelo teórico efetivo desenvolvido por Hudson and Liu (1999),
A)

\section{Plane distribution of small cracks}

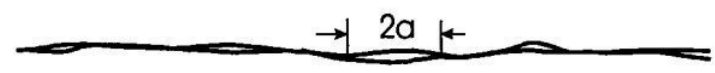

B)

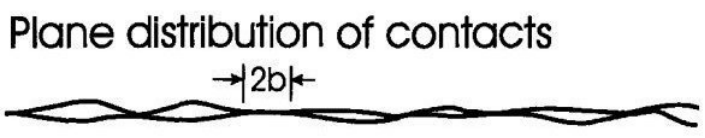

C)

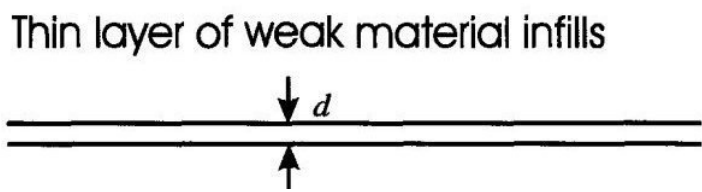

Figura 1: Representação ilustrativa dos modelos propostos por Hudson and Liu (1999).

suas características básicas e as diferentes aplicações do mesmo. O modelo proposto fornece uma formulação matemática baseada no processo de médias descritas em Schoenberg and Douma (1988) para as propriedades elásticas de rochas com simetria transversalmente isotrópica com eixo vertical.

\section{Metodologia}

No modelo efetivo proposto por Hudson and Liu (1999), as falhas são descritas individualmente como um conjunto de fissuras alinhadas preferencialmente e podem ser representadas por dois modelos distintos: Modelo 1, que descreve uma distribuição planar de fissuras circulares com diâmetro variável (Figura 1a), e o Modelo 2, que descreve uma distribuição planar de contatos circulares (Figura 1b). Ambos os modelos podem ser substituidos por uma camada equivalente, de espessura constante, preenchida por um material apropriado (Figura 1c).

Os coeficientes do tensor de rigidez elástica para o meio proposto por essa teoria são dados por: 


$$
\begin{aligned}
C_{11}=C_{22} & =\lambda+2 \mu & C_{33} & =\frac{\lambda+2 \mu}{1+E_{N}} \\
C_{12} & =\lambda & C_{13}=C_{23} & =\frac{\lambda}{1+E_{N}} \\
C_{44}=C_{55} & =\frac{\mu}{1+E_{T}} & C_{66} & =\mu
\end{aligned}
$$

Os valores En e Et dependem do modelo que está analisando. Para o caso do Modelo 1, En e Et são dados por:

$$
\begin{gathered}
E_{N}=\left(\frac{v^{s} a^{3}}{H}\right)\left(\frac{\lambda+2 \mu}{\mu}\right) U_{3}\left[1+\pi U_{3}\left(v^{s} a^{2}\right)^{\frac{3}{2}}\left(1-\frac{\mu}{\lambda+2 \mu}\right)\right] \\
E_{T}=\left(\frac{v^{s} a^{3}}{H}\right) U_{1}\left[1+\frac{\pi}{4} U_{1}\left(v^{s} a^{2}\right)^{\frac{3}{2}}\left(3-2 \frac{\mu}{\lambda+2 \mu}\right)\right]
\end{gathered}
$$

onde a é o raio das fissuras, $v^{s}$ é o número de densidade de fissura no plano de falha, $\mathrm{H}$ é o espaçamento entre os planos de falha e $\mu$ e $\lambda$ são os parâmetros de Lamè associados ao meio isotrópico. A densidade de fissura para este caso é dada por $\varepsilon=v^{s} a^{3} / H$. A área relativa de fissuramento, por sua vez, é dada por $r=v^{s} \pi a^{2}$. As fraturas associadas ao Modelo 1 podem ser substituidas por uma camada elasticamente equivalente com módulos de incompressibilidade e cisalhamento dados por:

$$
\begin{aligned}
& K^{*}=K^{\prime}-\frac{\pi \alpha \mu}{4}\left(\frac{\mu}{\lambda+2 \mu}\right)\left[1-\frac{4}{3}\left(\frac{r^{3}}{\pi}\right)^{1 / 2}\right] \\
& \mu^{*}=\mu^{\prime}+\frac{3 \pi \alpha \mu}{16}\left(\frac{3 \lambda+4 \mu}{\lambda+2 \mu}\right)\left[1-\frac{4}{3}\left(\frac{r^{3}}{\pi}\right)^{1 / 2}\right]
\end{aligned}
$$

onde $K^{\prime}$ e $\mu^{\prime}$ são os módulos de incompressibilidade e cisalhamento do material que preenche as fissuras.

Para o Modelo 2, os valores de En e Et são:

$$
\begin{aligned}
& E_{N}=\frac{(\lambda+2 \mu)^{2}}{4 \mu(\lambda+\mu)} \frac{1}{\left(v^{w} H b\right)}\left(1+2 \sqrt{v^{w} b^{2}}\right)^{-1} \\
& E_{T}=\frac{(3 \lambda+4 \mu)}{8(\lambda+\mu)} \frac{1}{\left(v^{w} H b\right)}\left(1+2 \sqrt{v^{w} b^{2}}\right)^{-1}
\end{aligned}
$$

onde $b$ é o raio da região de contato e $v^{w}$ é o número de densidade dos contatos no plano de fratura. Assim como no caso do Modelo 1, o plano de fissuras pode ser representado por uma camada elasticamente equivalente, cujos módulos de incompressibilidade e cisalhamento são dados por:

$$
\begin{gathered}
K^{*}=K^{\prime}-\frac{4 \mu(d / b) r(1-r)}{3 \pi} \frac{(\lambda+\mu)(4 \mu-\lambda)}{(3 \mu+4 \lambda)(\lambda+2 \mu)}\left[1+2\left(\frac{1-r}{\pi}\right)^{1 / 2}\right] \\
\mu^{*}=\mu^{\prime}-\frac{8 \mu(d / b) r(1-r)}{\pi}\left(\frac{\lambda+\mu}{3 \lambda+4 \mu}\right)\left[1+2\left(\frac{1-r}{\pi}\right)^{1 / 2}\right]
\end{gathered}
$$

onde $d$ é a abertura da área fissurada.

\section{Resultados e discussões}

Foi realizada a análise do comportamento das velocidades de ondas $\mathrm{P}$ e $\mathrm{S}$ e de seus parâmetros anisotrópicos, calculados a partir do modelo efetivo em estudo, para diversas situações geológicas. Os gráficos e mapas aqui apresentados foram feitos utilizando-se o software Matlab. Para todos os casos, utilizou-se como dados de entrada os parâmetros elásticos e petrofísicos da matriz isotrópica da rocha que queremos simular. A escolha foi simular arenitos, cujas propriedades da matriz foram definidas como as seguintes: $\mathrm{Vp}=3100 \mathrm{~m} / \mathrm{s}, \mathrm{Vs}=2300$ $\mathrm{m} / \mathrm{s}$, Densidade $=2200 \mathrm{~kg} / \mathrm{m}^{3}, \phi($ Porosidade $)=10 \%$. Foi simulado também o caso em que há saturação da rocha por água. Neste caso, além das propriedades da matriz isotrópica, utilizou-se como entrada as propriedades elásticas da água. São elas: $\mathrm{V} p=1500 \mathrm{~m} / \mathrm{s}$ e Densidade $=1000 \mathrm{~kg} / \mathrm{m}^{3}$.

Como dito anteriormente, o modelo efetivo de Hudson and Liu (1999) simula meios com simetria VTI. Portanto, as velocidades aqui estimadas e analisadas são aquelas associadas às ondas e aos modos de vibração existentes nesse tipo de meio. Foram estimadas as velocidades das ondas $\mathrm{P}$ que se propagam paralelamente (onda $\mathrm{P}$ rápida), perpendicularmente (onda $\mathrm{P}$ lenta) e diagonalmente (onda $\mathrm{P} 45^{\circ}$ ) ao plano de fraturamento. Quanto à onda $\mathrm{S}$, foram estimados os modos que vibram paralelamente (Onda $S$ rápida) e perpendicularmente (Onda $S$ lenta) ao plano de fraturamento. Para um melhor entendimento das ondas analisadas, a Figura 2 ilustra as direções de propagação e vibração dessas ondas em um meio altamente fraturado.

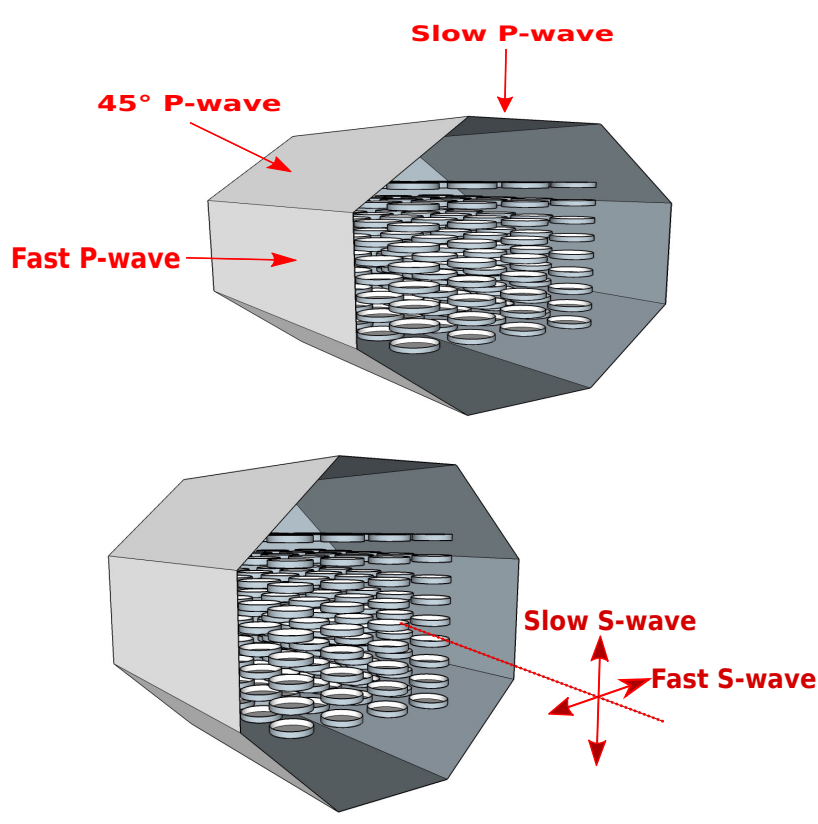

Figura 2: Representação ilustrativa de um meio altamente fissurado, semelhante ao proposto por Hudson and Liu (1999), mostrando as direções de propagação e vibração analisados neste trabalho.

As velocidades foram calculados a partir das seguintes relações: 


$$
\begin{gathered}
V_{P_{\text {fast }}}=\sqrt{C_{11} / \rho} \quad V_{P_{\text {slow }}}=\sqrt{C_{33} / \rho} \\
V_{P_{45^{\circ}}}=\sqrt{C_{11}+C_{33}+2 C_{44}+\sqrt{C_{11}-C_{33}^{2}}+4\left(C_{13}+C_{44}^{2}\right) / 4 \rho} \\
V_{S_{\text {fast }}}=\sqrt{C_{66} / \rho} \quad V_{S_{\text {slow }}}=\sqrt{C_{44} / \rho}
\end{gathered}
$$

onde $V_{P_{\text {slow }}}, V_{P_{45^{\circ}}}$ e $V_{P_{\text {fast }}}$ são, respectivamente, as velocidades das ondas $\mathrm{P}$ lenta, diagonal e rápida, $V_{S_{\text {fast }}}$ e $V_{S_{\text {slow }}}$ são, respectivamente, as velocidades de ondas $S$ rápida e lenta, $C_{i j}$ é o tensor de rigidez elástica e $\rho$ é a densidade da rocha, calculada em função de parâmetros da matriz isotrópica e da densidade de fissura da rocha através da seguinte relação:

$$
\rho=\left(1-\phi_{\text {total }}\right) \rho_{\text {iso }}
$$

em que

$$
\phi_{\text {total }}=\phi_{\text {iso }}+\phi_{\text {iso }}(1-\varepsilon)
$$

onde $\phi_{i s o}$ é a porosidade da matriz isotrópica, $\rho_{i s o}$ é a densidade da rocha isotrópica, $\varepsilon$ é a densidade de fissura e $\phi_{\text {total }}$ é a porosidade total das rochas fraturadas.

\section{Análise do Modelo 1}

As Figuras 3 à 7 mostram a variação das velocidades em função da densidade e do raio das fissuras, para o caso do Modelo 1. Foram analisadas densidades de fissura que variam de de $0 \%$ à $50 \%$, além de três valores de raio das fissuras: $3 \mathrm{~mm}, 4 \mathrm{~mm}$ e $5 \mathrm{~mm}$. As velocidades das ondas $P$ e $S$ rápidas (Figuras 3 e 6 ) mostram apenas uma pequena variação em função da densidade de fissura. Isso ocorre pelo fato de ambas as ondas se propagarem paralelamente ao plano de fraturamento, não sendo influenciadas diretamente pelas fissuras, e sim pela variação de densidade ocasionada pelo fraturamento do meio. Por outro lado, as velocidades das ondas P lenta, diagonal e $S$ rápida (Figuras 5, 4 e 7) variam tanto com a densidade de fissura quanto o raio das fissuras. Para todos os caso é verificado que, para uma mesma densidade de fissura, há uma maior diminuição na velocidade quanto menor é o raio da fissura. Isso evidencia que, para o modelo teórico aqui estudado, a quantidade de fissuras existentes influencia mais nas velocidades do que o raio das mesmas. Dentre todas as velocidades, a da onda $\mathrm{P}$ lenta é a mais afetada pelo fraturamente, constatado pela grande perda de magnitude no intervalo de densidade de fissura analisado. Além disso, a velocidade da onda $P$ lenta mostra sofrer uma variação ligeiramente maior devido a presença de fraturamento, já que apresenta uma mais rápida variação com o aumento da densidade de fissura se comparada com as outras velocidades.

A segunda análise é realizada a partir das Figuras 8 à 10, que representam mapas de velocidade em função do número de fissuras e de seus raios médios. Esses parâmetros e seus intervalos foram escolhidos por serem os mais comumente controlados em um procedimento de modelagem física, o que será um complemento futuro ao

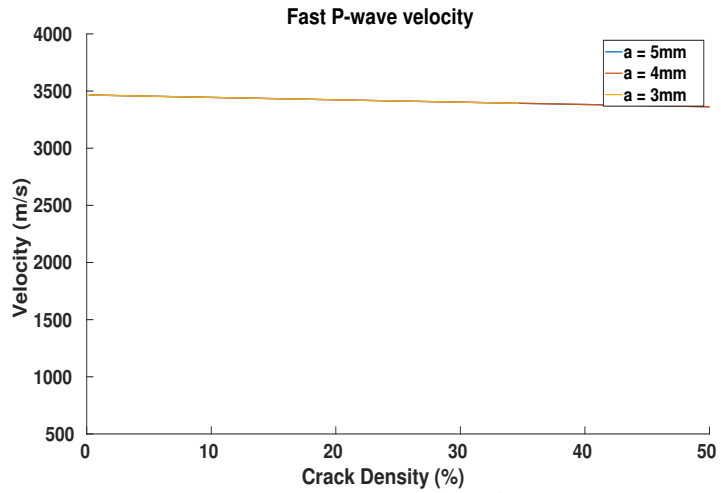

Figura 3: Velocidade da onda $P$ rápida em função da densidade de fissuras para três valores de raios das fissuras.

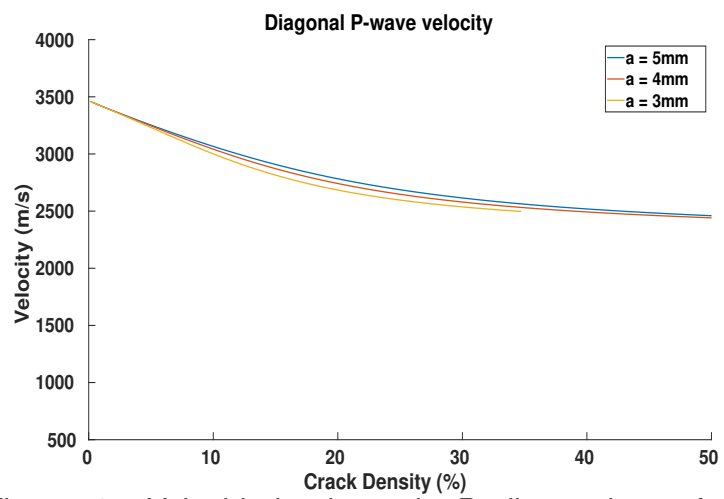

Figura 4: Velocidade da onda $P$ diagonal em função da densidade de fissuras para três valores de raios das fissuras.

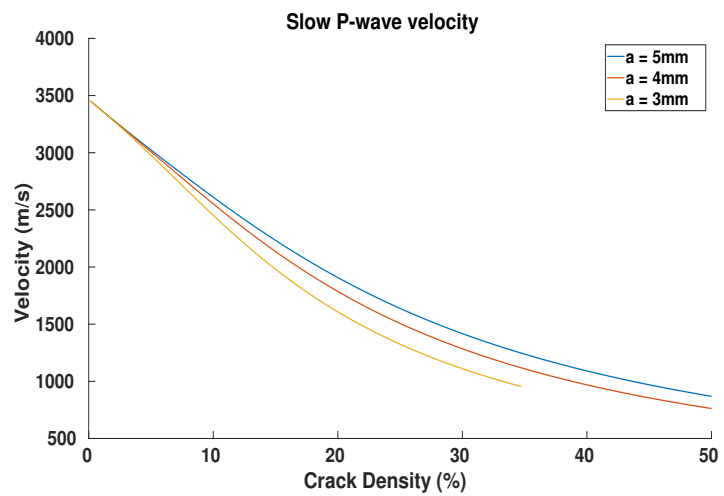

Figura 5: Velocidade da onda $P$ lenta em função da densidade de fissuras para três valores de raios das fissuras.

presente trabalho e será mais discutido $\mathrm{m}$. A análise dos mapas mostra que a velocidade da onda P lenta é também a mais afetada com a variação da quantidade de fissura e do raio médio das mesmas. É evidente também que as velocidade das ondas $P$ e $S$ lentas sofrem uma maior e mais forte variação vertical do que horizontal, o que corrobora com a nossa interpetação anterior que o número de inclusões tem maior influência na velocidade que o raio das mesmas.

As Figuras 11 à 13 mostram a variação das velocidades 

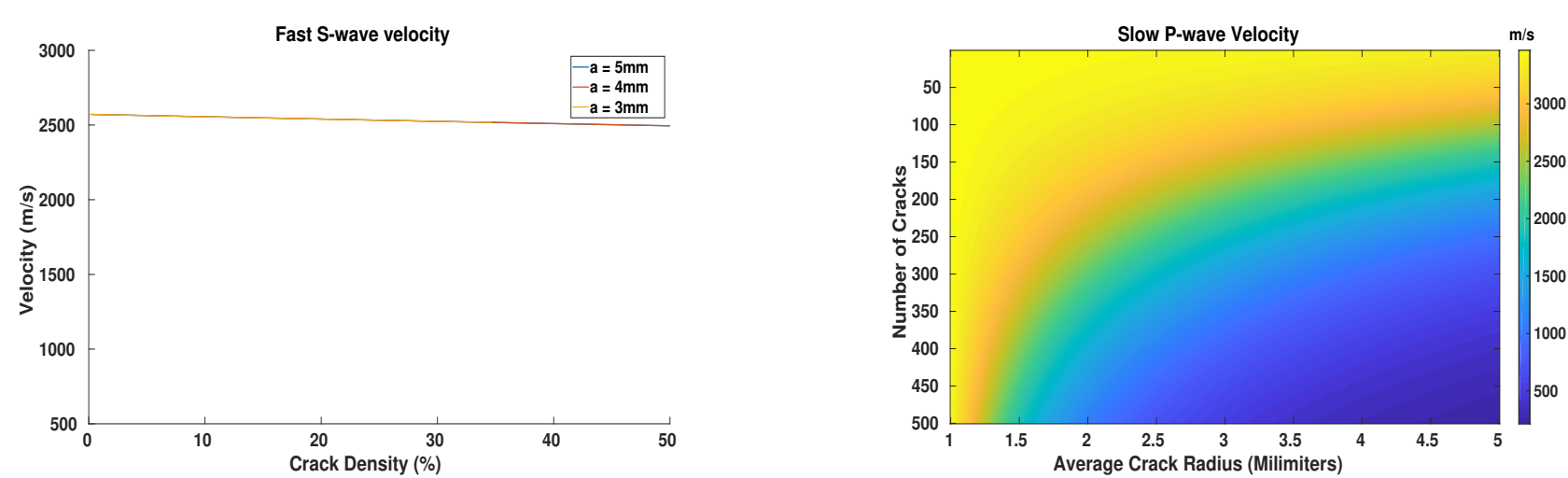

Figura 6: Velocidade da onda S rápida em função da densidade de fissuras para três valores de raios das fissuras.

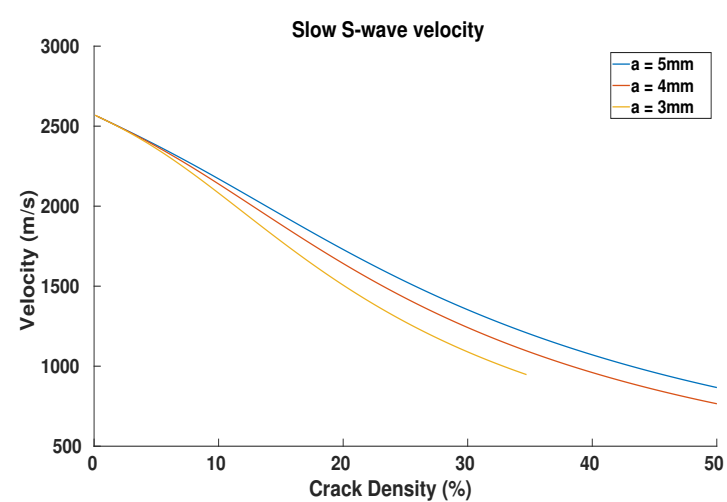

Figura 7: Velocidade da onda S lenta em função da densidade de fissuras para três valores de raios das fissuras.

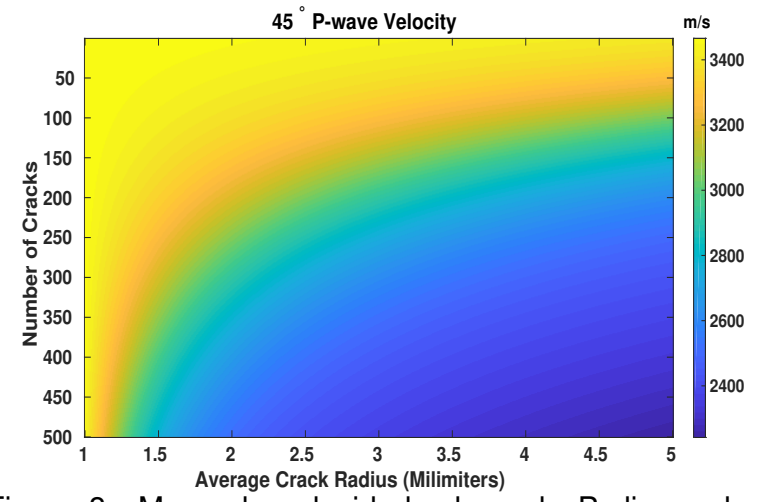

Figura 8: Mapa de velocidade da onda $P$ diagonal em função do número de fissuras e do raio médio destas.

de ondas $\mathrm{P}$ lenta e diagonais e $\mathrm{S}$ lenta com a densidade de fissura para os casos em que as fissuras estão secas e saturadas, considerando o raio médio das fissuras igual a $5 \mathrm{~mm}$. Apenas essas velocidades são analisadas porque foram as que se mostraram mais suscetíveis a influência do fraturamento. A velocidade da onda P lenta é altamente influenciada pela saturação, sendo visível uma grande variação em sua magnitude em comparação com o caso da rocha seca. A velocidade da onda $P$ diagonal, por sua vez, mostra apenas uma pequena variação entre os dois casos, até a densidade de fissura de $10 \%$. A velocidade

Figura 9: Mapa de velocidade da onda $P$ lenta em função do número de fissuras e do raio médio destas.

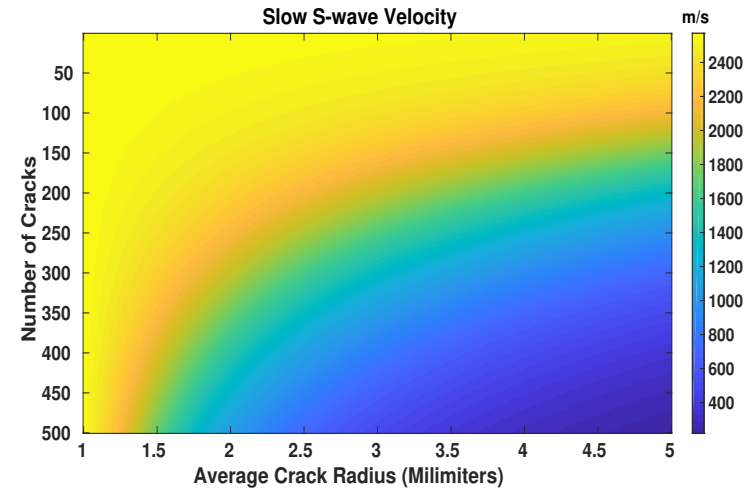

Figura 10: Mapa de velocidade da onda S lenta em função do número de fissuras e do raio médio destas.

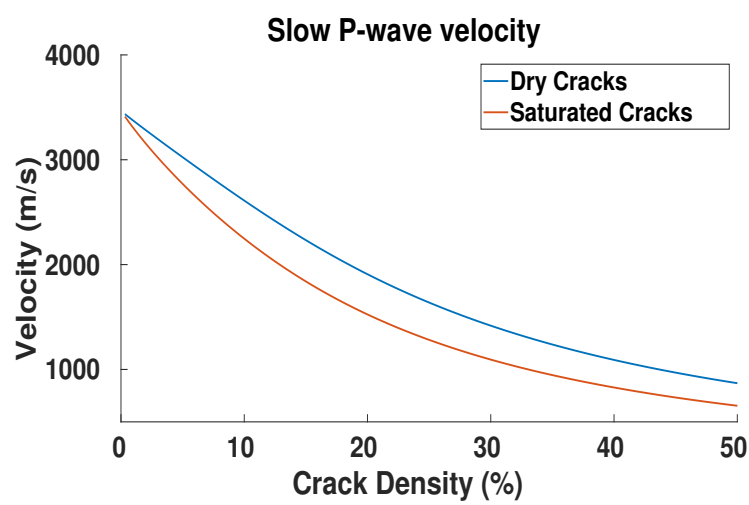

Figura 11: Velocidade da onda $P$ lenta em função da densidade de fissuras nas condições seca e saturada por fluido.

de onda $S$, como era esperado, não mostra variação de velocidade para os dois casos. Isso ocorre porque a onda S não se propaga no fluido, não sofrendo influência nem do ar nem da água.

\section{Análise do Modelo 2}

Os mapas das Figuras 14 à 16 referem-se à variação das velocidades de ondas $P$ e $S$ lentas e onda $P$ diagonal em função do número e do raio médio dos contatos 


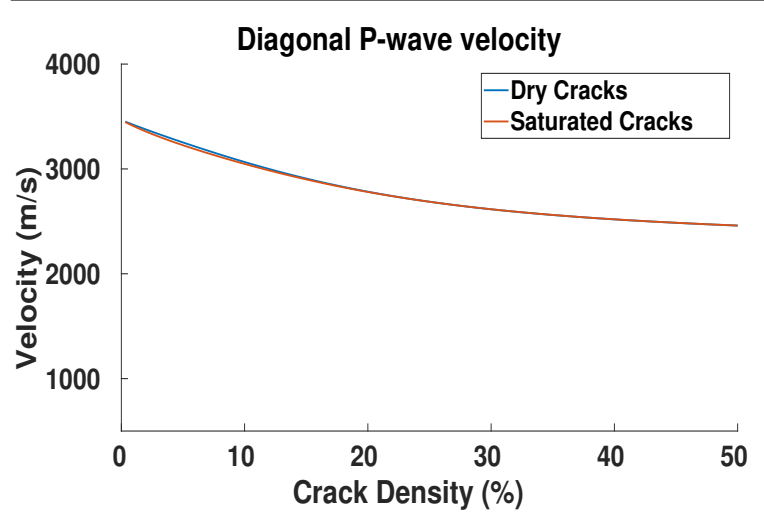

Figura 12: Velocidade da onda $P$ diagonal em função da densidade de fissuras nas condições seca e saturada por fluido.

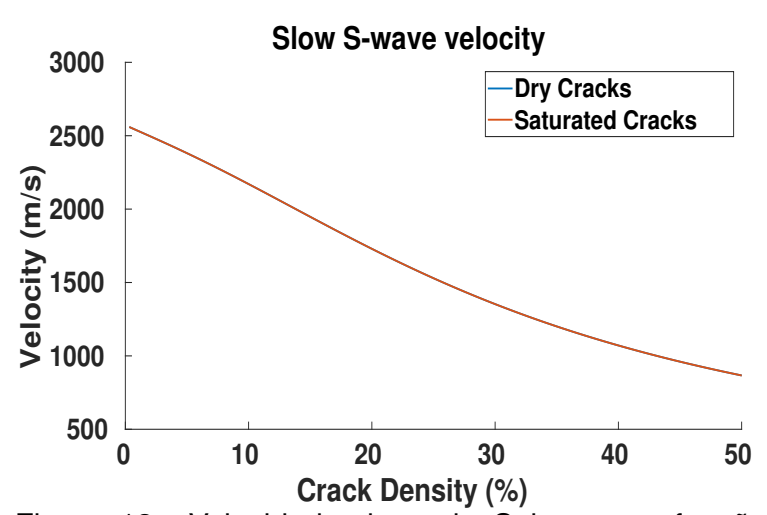

Figura 13: Velocidade da onda $S$ lenta em função da densidade de fissuras nas condições seca e saturada por fluido.

existentes na rocha. Contato refere-se ao afastamento existente entre fissuras que possuem o mesmo diâmetro e seu raio é um parâmetro que influencia diretamente na densidade de fissura do meio. Apenas as velocidades lentas e a $\mathrm{P}$ diagonal foram analisadas pois são as únicas influenciadas diretamente pelo fraturamento, como visto na análise do Modelo 1. Para todos os casos, as velocidades possuem um comportamento diferente do presente nos mapas analisados previamente, para o Modelo 1 . No caso do Modelo 2, as menores velocidades localizam-se na extremidade esquerda dos mapas, enquanto que para o Modelo 1 localizam-se na extremidade direita. Um raio de contato pequeno significa que o meio possui a maior parte de sua superfície fissurada, o que acarreta em alta densidade de fissura, o que explica esse deslocamento das menores velocidades para a esquerda. Semelhante ao analisado no caso do Modelo 1, o número de contatos tem maior influência na velocidade do que o raio, explicado pela maior variação vertical do que horizontal de velocidade observada nos mapas. Nota-se também que as velocidades pouco variam a partir do raio de contato de $2 \mathrm{~mm}$. Uma explicação para isso é que, a partir desse valor, o meio deixa de ser altamente fraturado, fazendo com que os cálculos utilizando o Modelo 2, que prevê meios fortemente fraturados, passem a ser inexatos ou imprecisos.

Para se realizar uma análise mais minuciosa do que foi afirmado no parágrafo anterior, foram gerados gráficos da

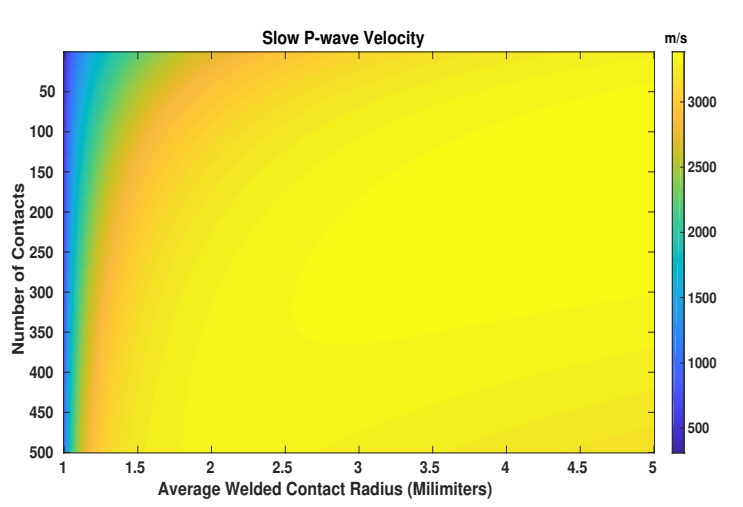

Figura 14: Mapa de velocidade da onda $P$ lenta em função do número de fissuras e do raio de contato médio destas.

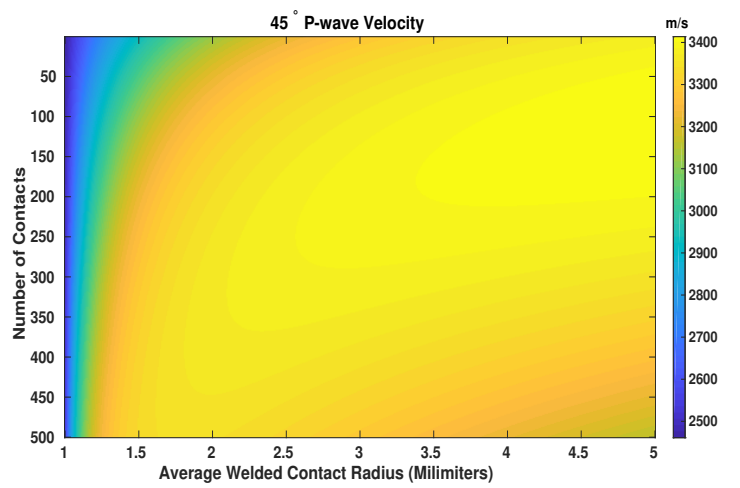

Figura 15: Mapa de velocidade da onda $P$ diagonal em função do número de fissuras e do raio de contato médio destas.

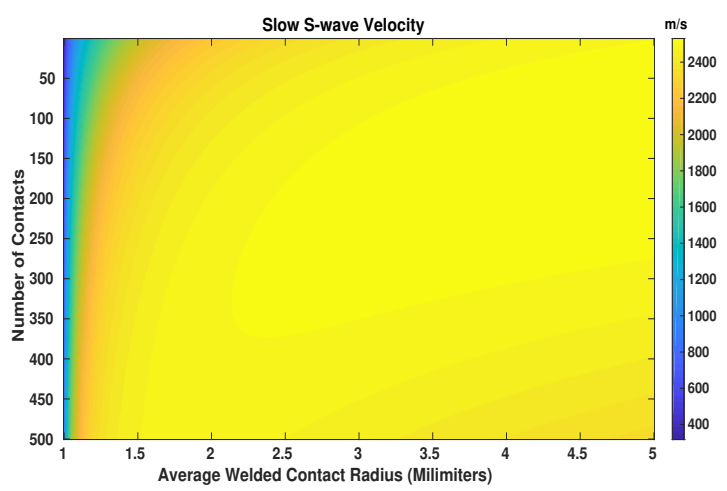

Figura 16: Mapa de velocidade da onda S lenta em função do número de fissuras e do raio de contato médio destas.

variação das velocidades em função do raio médio dos contatos, considerando diferentes números de contatos. Os gráficos são mostrados nas Figuras 17 à 19 . É visível para todas as velocidades analisadas nas figuras abaixo que o menor valor é associado ao menor raio de contato, ou seja, ao meio mais fraturado. Isso já tinha sido constatado na análise dos mapas anteriormente apresentados. Porém, o que não era visível nos mapas, é que, em um certo ponto, as velocidades fogem do padrão esperado e têm sua magnitude diminuída. Esse ponto de reversão de comportamento varia para cada velocidade e para cada valor de número de contatos, sendo maior 


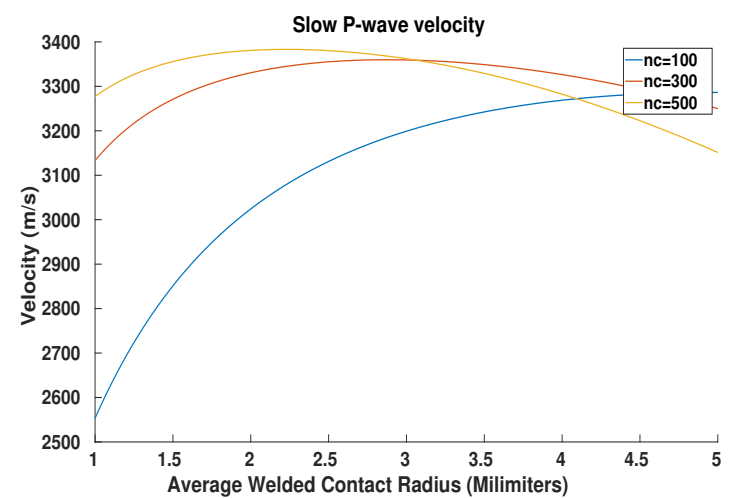

Figura 17: Velocidade da onda $P$ lenta em função do raio de contato médio das fissuras para três diferentes números de contatos.

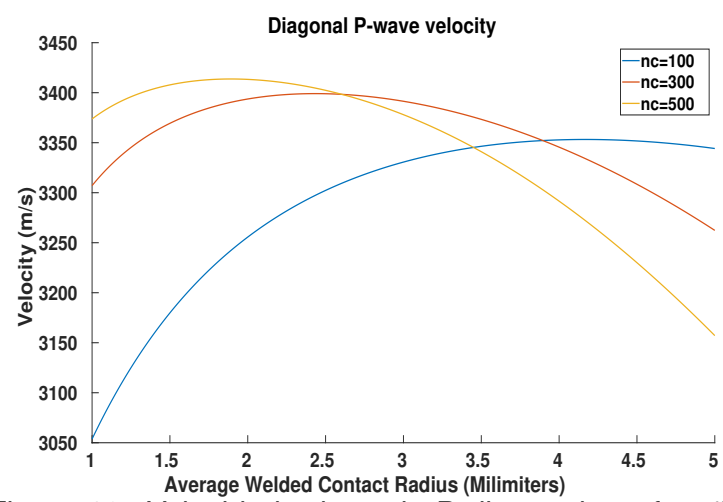

Figura 18: Velocidade da onda $P$ diagonal em função do raio de contato médio das fissuras para três diferentes números de contatos.

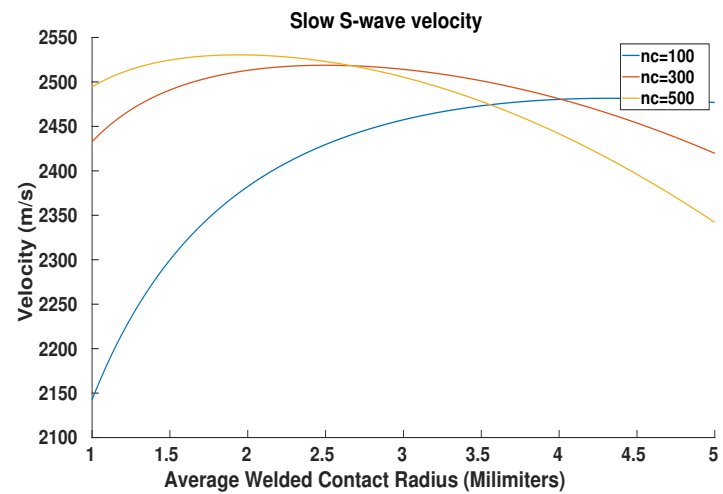

Figura 19: Velocidade da onda S lenta em função do raio de contato médio das fissuras para três diferentes números de contatos.

quanto menor for o número de contatos existente na rocha. Apesar de esse comportamento não ter sido observado com clareza nos mapas, sua análise corrobora com o que foi observado anteriormente, de que o Modelo 2 proposto por Hudson and Liu (1999) tem um limite para sua aplicação confiável.

\section{Conclusões}

A partir da análise dos resultados associados ao Modelo 1 , foi visto que as velocidades das ondas $P$ e $S$ rápidas não sofrem influência das fissuras, sendo unicamente influenciadas pela densidade total do meio. A velocidade da onda $P$ lenta, dentre todas, foi a que mostrou maior variação com o aumento da densidade de fissura, enquanto a onda $P$ diagonal mostra uma variação bem mais discreta. Viu-se também que a quantidade de fissuras existentes no meio tem uma maior influência nas velocidades que seus raios. A saturação diminui consideravelmente a velocidade da onda $\mathrm{P}$ lenta, enquanto que influencia apenas até a densidade de fissura de $10 \%$ para a velocidade de onda $\mathrm{P}$ diagonal.

Para o Modelo 2, as velocidades têm seus menores valores quando o valor do raio dos contatos é menor. Isso ocorre pelo fato de um baixo valor de raio acarretar em meio com pouca quantidade de áreas não fraturadas. Apesar dessa influência, foi visto que o número de contatos possuem maior influência nas velocidades do que seus raios. A existência de uma mudança no comportamento das velocidades em função do raio dos contatos, verificou-se que o Modelo 2 possui limites para sua aplicação confiável, limite este que depende do número de contatos existente na rocha e do tipo de onda analisada.

Futuramente, como parte experimental desse trabalho, pretende-se construir amostras em laboratório que simulem o meio VTI nas condições de fraturamento dos modelos 1 e 2. Assim, pode-se comparar os resultados obtidos através dos modelos teóricos de Hudson and Liu (1999) com medidas experimentais, e por conseguinte, verificar o quão próximo da realidade essa teoria de meios efetivos se encontra.

\section{Referências}

Hudson, J., and E. Liu, 1999, Effective elastic properties of heavily faulted structures: GEOPHYSICS, 64, 479-485.

Hudson, J. A., 1980, Overall properties of a cracked solid: Mathematical Proceedings of the Cambridge Philosophical Society, 88, 371-384.

- 1981, Wave speeds and attenuation of elastic waves in material containing cracks: Geophysical Journal of the Royal Astronomical Society, 64, 133-150.

Nelson, R. A., 1985, Geologic analysis of naturally fractured reservoirs: Gulf Pub. Co., Book Division.

Sayers, C., 2007, Seismic characterization of reservoirs containing multiple fracture sets, in SEG Technical Program Expanded Abstracts 2007: Society of Exploration Geophysicists, SEG Technical Program Expanded Abstracts, 1456-1460.

Schoenberg, M., and J. Douma, 1988, ELASTIC WAVE PROPAGATION IN MEDIA WITH PARALLEL FRACTURES AND ALIGNED CRACKS1: Geophysical Prospecting, 36, 571-590. 